\title{
The parietal association cortex of the rat
}

\author{
FERNANDO TORREALBA ${ }^{1}$ and JOSÉ LUIS VALDÉS ${ }^{2}$.
}

\footnotetext{
${ }^{1}$ Departamento de Fisiología, Facultad de Ciencias Biológicas, Pontificia Universidad Católica de Chile, Santiago, Chile.

2 Departamento de Fisiología y Biofísica, Instituto de Ciencias Biomédicas, Universidad de Chile.
}

\begin{abstract}
Spatial cognition is a complex higher function in mammals and is involved in a variety of tasks that can be explored in the laboratory. In this review we will discuss the role of the posterior parietal/anteromedial cortex of rodents, also known as the parietal association cortex, and the hippocampal formation in spatial navigation. We will also discuss other higher associational functions of the posterior parietal/anteromedial cortex as they relate to Dr. Pinto-Hamuy's contribution to understanding behavioral functions.
\end{abstract}

Key terms: Association cortex, spatial navigation, visual cortex.

\section{INTRODUCTION}

Many important life events happen in a particular place and time, so that the recollection of those events is often associated with a more or less detailed mental reconstruction of places. The remembrance of those past individual life events uses a form of memory described as declarative memory, which may be a semantic memory if one vaguely recognizes the event as familiar, or an episodic memory when the recall belongs to a particular episode, and therefore the memories are much more accurate.

It is clear that in the recollection of an important life episode many brain systems participate in a distributed representation of the event (Byrne et al., 2007). The present state of understanding of brain functions indicates that particular brain systems play distinct roles in an episodic recollection. An important life event probably engages most of the following brain systems: one or more exteroceptive sensory system; spatial processing networks; the limbic system involved in processing emotionally relevant responses; and the executive areas of the prefrontal cortex (Hok et al., 2005).
Numerous functional and neuroanatomical studies have made it clear that many of the above mentioned systems are reciprocally interconnected and function in concert. We will discuss below the evidence linking the posterior parietal/anteromedial cortex to these central circuits, and we will also discuss some of the functional and behavioral studies relevant to spatial cognition in rodents. A recent article reviews the evidence, mostly from studies in humans, for the involvement of the parietal cortex in episodic memory (Cabeza et al., 2008).

In rats, the cortical region sandwiched between the primary auditory, somatosensory and visual cortices comprises 2 areas that can be distinguished based on cyto- and myeloarchitectonics (Fig. 1A): rostral Oc2M and ParP (Palomero-Gallaher and Zilles, 2004). There are several reasons to consider this cortical region, the posterior parietal/anteromedial cortex, as a multimodal association cortex quite comparable in location, functions and connections to the parietal association cortex of primates (Chen et al., 1994a; Kolb et al., 1994). Pioneering work led by Pinto-Hamuy (Pinto-Hamuy et al., 1987) first demonstrated the multimodal association 
nature of the posterior parietal/anteromedial cortex in rats, using a behavioral approach. Dr. Pinto-Hamuy engaged a number of colleagues with skills complementary to hers to understand the role of parietal and occipital cortical areas in solving different spatial tasks (Sanchez et al., 1997; Espinoza et al., 1999; Pinto-Hamuy et al., 2004; Espina-Marchant et al., 2006).

Spatial cognition and memory. To navigate in space, rats use allothetic and idiothetic (or egocentric) cues for guidance. The former cues come from the environment, while idiothetic clues are provided by the self- movement of the individual and are represented in the vestibular, propioceptive and somatosensory systems; idiothetic clues also may come from corollary copies of motor commands and from optic flow and other movement related information (Save and Poucet, 2005; Wallace and Whishaw, 2005).

A large number of errands a normal rodent carries out during a typical day involve the use of accurate and updated spatial information. Rats search for food
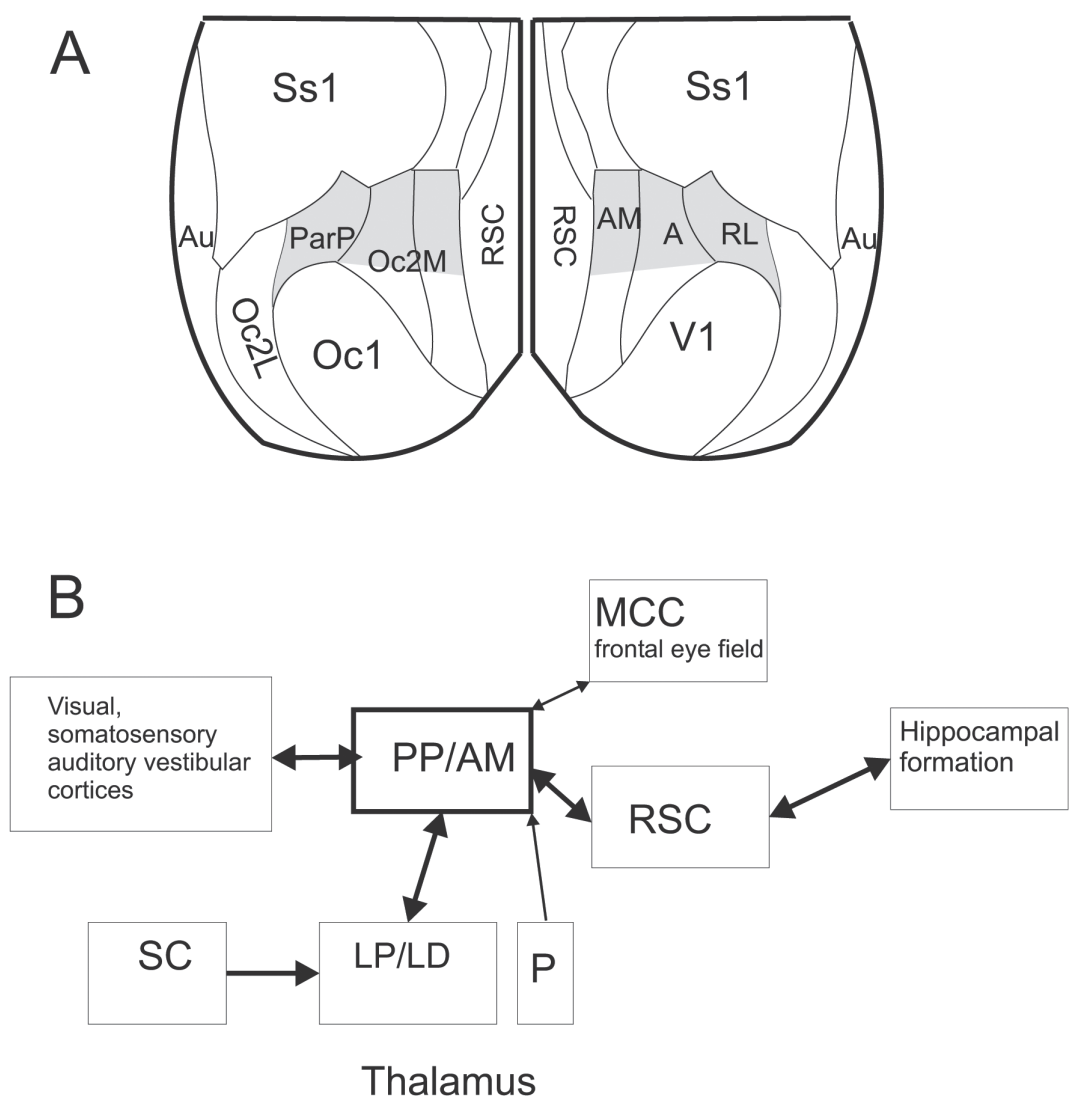

Figure 1: Schematic illustration of the layout and principal connections of the posterior parietal association cortex of rats. A. Dorsal view of the caudal half of the cortex showing at the left the cytoarchitectonic names (Palomero-Gallaher and Zilles, 2004) of areas forming the posterior parietal/anteromedial cortex of the rat (in gray, and indicated as PP/AM in B). At the right, the names of the visual areas forming this association cortex, as defined in the text. B. Major neuroanatomical connections of the posterior parietal/anteromedial area. Abbreviations: A, anterior visual area; AM, anteromedial visual area; $\mathrm{Au}$, auditory cortex; LP/LD, lateroposterior and laterodorsal thalamic nuclei; MCC, mid-cingulate cortex; Oc1 and V1, primary visual cortex; Oc2M, medial peristriate occipital cortex; Oc2L, lateral peristriate cortex; $\mathrm{P}$, posterior thalamic nucleus; ParP, posterior parietal cortex; PP/AM, posterior parietal/anteromedial cortex; RSC, retrosplenial cortex; RL, rostral lateral visual area; SC, superior colliculus. 
from a home base, often one of the entrances to the underground galleries they build. They store food for later consumption or materials to construct nests for pups and parents. The strategies rats use to explore the surroundings of their underground homes in search of food and nesting material have been studied in wellcontrolled experiments in the laboratory (Save and Poucet, 2005; Wallace and Whishaw, 2005).

These studies have shown that rats take outward (from home) trips that are radically different from the sprints back home. Outward excursions can take any of a number of circuitous routes until the food reward is found. In contrast, the trip back home is a beeline run at much higher speed than the outward, searching trips. So, animals should keep an internal representation of space, they know where they are and continuously update that information as they move, and also keep a representation of the goals they seek and of the starting or home place.

Hippocampus and place cells. O'Keefe and Dostrovski (O'Keefe and Dostrovsky, 1971) demonstrated that neurons in the hippocampus show place-dependent firing activity when the animal is exploring a spatial environment. The location where these "place cells" discharge action potentials is commonly known as the "place field". The population of place cells generates a complete representation of the space, a "spatial map" or "cognitive map". This concept of a cognitive map, along with previous evidences from lesion studies (O'Keefe, 1978) support the idea that hippocampal formation is absolutely necessary for navigation and for the storing of episodic memory related to location. This kind of evidence positions the hippocampus as a top level structure for understanding how space is represented in the brain. It is currently proposed that the internal navigational system per se in mammals is sufficient for the animal to navigate a particular space without external or allocentric cues.

The cognitive map in the hippocampus emerges from the conjunction of the "place cells" in the hippocampus coding for the location of the animal, the "head direction cells" located in the related thalamic region, coding for the orientation of the animal's head (Wiener and Taube, 2005), and the "grid cells" of the medial entorhinal cortex, which generate a coordinate map of the location where the animal can navigate (Hafting et al., 2005). These structures and those neurons act as a system that can determine the velocity at which the animal is moving from a specific starting place. This function probably relies on the theta wave oscillations recorded when the animal is navigating (Whishaw and Vanderwolf, 1973).

When the animal is navigating, this spatial map must be updated and a new representation for a new space must emerge. The way in which the place, grid and head direction cells modify activity to generate this new map is termed path integration (McNaughton et al., 2006). It is proposed that path integration is dependent on idiothetic, or self-motion, information such as motor efferent copy, vestibular information and visual outflow.

Even though the internal navigational system can be updated by a path integrator and this may be good enough for navigation, external cues like visual or other sensory modality landmarks can modify the cognitive map and can reorient the activity of the place cells. The typical evidence comes from experiments where the animal is navigating an arena within a room with visual cues. After the animal learns this space and the cognitive map is stable, it no longer needs the visual cue to navigate correctly. The place field of each place cell is stable across time, even in darkness, indicating the presence of an internal navigational system. However, if the experimenter rotates the visual cue by $90^{\circ}$ and then re-exposes the animal to this environment, the entire cognitive map rotates $90^{\circ}$. This indicates a clear influence of the external cue over the navigational system of the animal (Muller and Kubie, 1987; Quirk et al., 1990). As well, internal signals, like goals or the saliency of determinate locations, can modify the activity of the place cells. For example, when one small location where the rat is 
moving offers a reward, the place fields of the place cells tend to accumulate over that region (Hok et al., 2007).

Any change in position, frequency, or presence of the place fields in response to sensory information is called "remapping". The fact that the hippocampal formation is sensitive to remapping in response to sensory information leads to the need to understand how spatial and non-spatial information arrives to the hippocampus. A series of cortical structures, principally those that belong to the para-hippocampal formation such as the medial and lateral entorhinal cortex, perirhinal cortex and other high level visual cortices, are key components for bringing information to the hippocampus.

Entorhinal cortex: the principal gate of information to the hippocampus. Early evidences (Miller and Best, 1980) showed that lesions of the entorhinal cortex generate a strong drop off in the number of hippocampal cells with positional correlates in a radial maze spatial task. This finding suggests that the entorhinal cortex could be a key structure in the generation of place fields in the hippocampus and hence the formation of spatial maps.

The entorhinal cortex, defined as area 28 by Brodman, is the major external input to the hippocampal formation, (Fig. 2) and is considered an interface between the neocortex and the hippocampal formation (Kerr et al., 2007). This structure, located in the most caudal, ventral and lateral portion of the brain, is subdivided, based on its anatomical connections, in a medial and a lateral region. The lateral entorhinal cortex receives information principally from insular, piriform and frontal cortical regions, and from the amygdala and olfactory structures. The medial entorhinal cortex (MEC) receives information from visual, posterior parietal and retrosplenial cortices and from midline, dorsolateral and dorsomedial thalamic nuclei (Kerr et al., 2007). So, MEC is the anatomical interphase between the posterior parietal/ anteromedial cortex, mostly via the retrosplenial cortex, and the hippocampus (Fig. 1B).

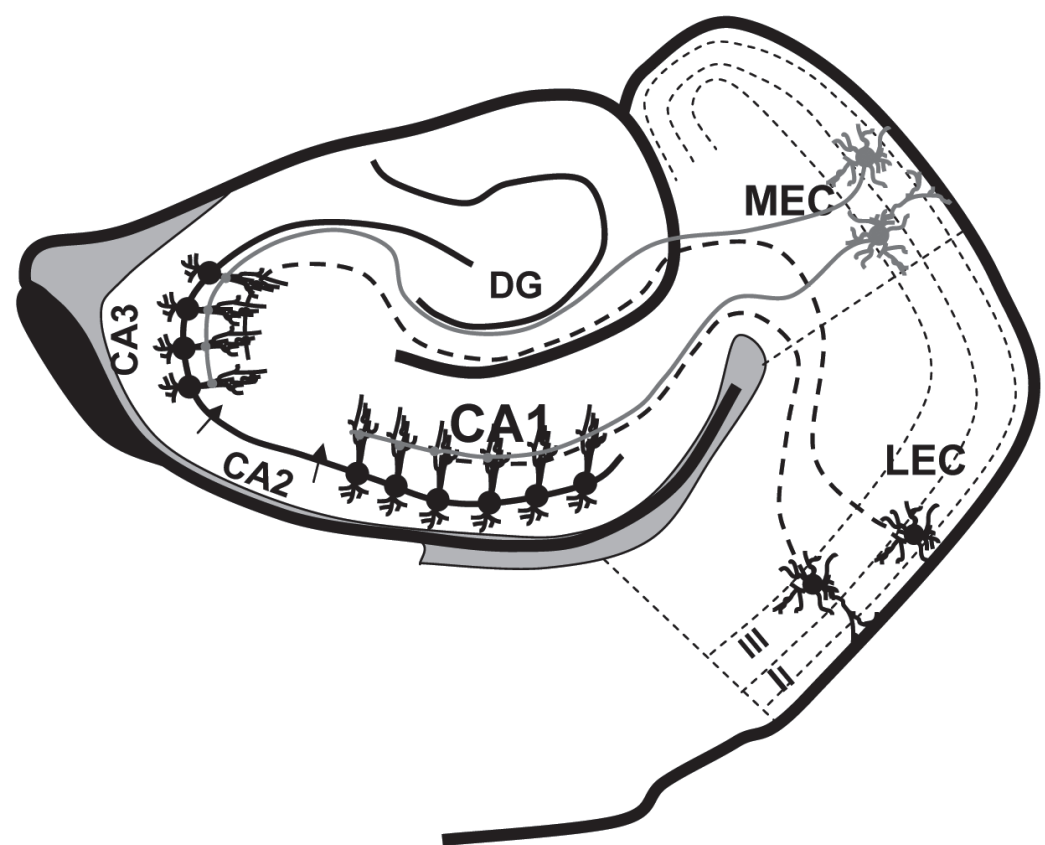

Figure 2: Schematic illustration of the principal connections from the lateral entorhinal cortex (LEC) and medial entorhinal cortex (MEC) to the apical dendrites of the hippocampal pyramidal neurons in CA1 and CA3. D.G., dentate gyrus; II y III indicate layers of the entorhinal cortices. 
The entorhinal cortices send projections principally to the hippocampal formation (Steward and Scoville, 1976; Steward, 1976; Witter and Amaral, 2004). The fibers coming from layer II of MEC terminate at the mid proximo-distal portion of the apical dendrites of CA3 pyramidal neurons, while the fibers coming from layer III end in the region of CA1 close to CA2 (proximal CA1). The axons from the lateral entorhinal cortex end at the distal apical dendrite of CA3 neurons and in the region of CA1 close to the subiculum (distal CA1, see Fig.1). This special array of connections suggests a separate entry of external information, at least to CA1. The question is if those two entorhinal structures are processing different information or not.

The work of Moser's group showed that neurons on the MEC have multiple, discrete and stable place fields where those neurons discharge (Fyhn et al., 2004). Those MEC firing fields are still present after the hippocampus is damaged, suggesting that MEC may be centrally involved in spatial processing. A new set of players came into the picture when the same group (Hafting et al., 2005) demonstrated in MEC the existence of the "grid cells", neurons that have a different form of spatial tuning. This population of neurons form a topographical spatial map of the environment represented as a grid tessellating equilateral triangles over the entire spatial environment. These evidences suggest an important role for MEC in processing spatial information.

Lateral entorhinal cortex neurons in contrast have less spatial specificity. The pattern of afferents that this structure receives from the insular cortex, olfactory structure, amygdala and perirhinal cortex (a polymodal sensory processing region) suggests that it may play a role in providing non-spatial information about context and emotional significance (Kerr et al., 2007).

Even though advances in the understanding of the function of the hippocampal formation and parahippocampal structures have been substantial, we still have many questions about how information is coming through those regions. Probably one of the most important sensory modalities that contribute to the spatial navigational system is the visual system. In addition to contributing to the internal representation of space through the retinotopic maps present in many visual cortical areas, the visual system provides information about optical outflow, which indicates self-movement (Schaafsma and Duysens, 1996) during navigation and is therefore necessary for path integration. The visual system is probably one of the systems that more easily affect the spatial map, (i.e. visual cues rotations). One of the few structures from the visual system that has an important role in solving spatial tasks is the anteromedial extrastriate visual cortical area. This area was a focus of attention of the Dr. Pinto-Hamuy (PintoHamuy et al., 1987).

The parietal association cortex: Several tests have been devised for laboratory use in studying the neural and behavioral mechanisms rats use to navigate. Among the simplified tasks used to test learning and memory involving spatial information, the most common tests occupy different types of mazes (Espinoza et al., 1999; Pinto-Hamuy et al., 2004). The learning of laboratory tasks that involve spatial memory is then a valid and simplified model of particular strategies that rats use in their natural lives to search for and retrieve nesting material, food or simply to explore new surroundings. Area PP/AM is required when working with previous information and long-term memories are needed to efficiently gather food in a laboratory model of foraging behavior (Espina-Marchant et al., 2006).

It has long been known that injury to the posterior parietal cortex of rats impairs the acquisition and retention of maze habits, as discussed by McDaniel (1985). He pointed out that lesion to this parietal cortex also impairs learning successive spatial reversals (McDaniel, 1985). As mentioned above, a pioneering behavioral study by PintoHamuy and colleagues (1987), where lesion to the anteromedial extrastriate visual cortex prevented the acquisition of a conditional visuosomatic response, indicated that this area was better considered as a multimodal association 
cortex. A further analysis of behavioral deficits after area AM lesion showed that this visual area was important to learn a maze habit, even in blind rats (PintoHamuy et al., 2004), in contrast to lesions to the lateral extrastriate visual areas where a lesion impaired the learning of visuovisual associations (Gallardo et al., 1979).

The anteromedial extrastriate visual cortical area (area AM) is located at the rostral end of the medial extrastriate visual cortex (Fig. 1A). This cortical area can be considered an associative cortical area for several reasons. It receives a topographic projection from the primary (striate) visual cortex, as is the case for the lateral extrastriate visual areas in cortical area Oc2L (Montero, 1993). Area AM contains a complete representation of the contralateral visual hemifield (Montero et al., 1973b), but unlike the striate cortex, it has no overrepresentation of the central visual field, but both peripheral and central visual fields are equally represented. This linear representation of the visual field seems appropriate to map visual space without the bias towards the central visual field present in other visual areas.

From a functional perspective, visual area AM actually belongs to a cortical association area involved in spatial tasks. This area includes parietal and occipital cortices that process allocentric as well as egocentric signals (see below) necessary for navigation in space, and are involved in other tasks that require the association of visual and somatosensory cues. From a network perspective, visual area AM may be considered as part of the dorsal (towards the parietal cortex) stream of visual processing described in primates, and lateral extrastriate visual areas as part of a ventral (towards the temporal region) visual stream.

There is not yet consensus on the parcellation of the posterior parietal/ anteromedial cortex. However, this is an area of relatively homogeneous cytoarchitectonics, as has been commented before (Kolb, 1990; Palomero-Gallaher and Zilles, 2004) and as we have observed in our preparations. This cortical association area is located among unimodal primary sensory cortices, which have much more differentiated lamination and more myelin, making them very distinct in the Nissl- and myelin-stained sections normally used to assess the extent of lesions or cannulae placement in behavioral studies based on neuronal inactivation, or the precise recording places in electrophysiological studies. These cortices are the primary visual area caudally, and the primary somatosensory cortex rostrally in respect to the posterior parietal/anteromedial cortex. While cytoarchitectonic analysis cannot reliably make a distinction between areas within the posterior parietal/anteromedial cortex, myeloarchitectonic analysis can provide such a distinction. Decreased myelin in the intermediate layers of Oc2M (Montero, 1993, Figs 1 and 6) and more myelin (Kolb, 1990) in the more lateral parietal areas (ParPD and ParPR, Figs. 11 and 14 of Palomero-Gallaher and Zilles, and formerly the rostral extension of OC2L in (Zilles, 1990)) differentiate these two regions.

Functional studies have provided evidence for the existence of more cortical areas than architectonic studies. Detailed electrophysiological studies on the visual responses in the peristriate cortex, which have been for the most part analysis of retinotopic visual maps, complemented with careful studies of the connections made by the areas defined by their retinotopy, have identified several distinct areas in this cortical association region in the posterior parietal/anteromedial cortex.

Retinotopical organization of the posterior parietal/anteromedial cortex. The first detailed study of the rat visual cortex, based on the electrophysiological responses of single neurons to moving visual stimuli (Montero et al., 1973b), demonstrated the existence of multiple visual areas in the posterior cortex, each area with a more or less complete representation of the contralateral visual hemifield. This study suggested that, in addition to the already known primary visual area (striate cortex or area 17), there are five other visual cortical areas that surround the primary visual cortex. In this study and in a complementary paper on the efferent 
connections of the striate cortex (Montero et al., 1973a), the authors tentatively described two visual areas within what we are now calling the posterior parietal/ anteromedial cortex. One area was placed anteromedial and the other was anterior to the striate cortex. However, the confirmation of distinct visual areas rostral to the primary visual cortex came from ulterior studies that combined retinotopical mapping with connectional criteria in the same animal (Espinoza and Thomas, 1983; Thomas and Espinoza, 1987; Montero, 1993). Presently, 3 retinotopically distinct areas have been defined in the posterior parietal/anteromedial cortex, and for them we applied here the nomenclature of Montero (1993), where he re-analyzed the retinotopy of the connections between striate and extrastriate cortical areas to clarify and summarize the previous studies. The cortical areas within the posterior parietal/anteromedial cortex are: area anteromedial (AM), the anterior area (A) and the rostral lateral area (RL), (Fig. 1A) each with a representation of the contralateral visual hemifield. Area AM is located in the anatomically defined rostral part of Oc2ML (Montero, 1993, Zilles, 1985), while area A corresponds to rostral Oc2MM and area RL is located in the rostral and medial part of Oc2L (or ParP).

The associational nature of the posterior parietal/anteromedial cortex is also shown in electrophysiological recordings in freely moving rats. In this cortex neurons have been found that code for head direction in the absence of visual cues or in darkness (Chen et al., 1994a), cells that code mnemonically for directional movement and spatial representations on the basis of visual cues (Chen et al., 1994b), and cells that code mnemonically for spatial representation on the basis of acoustic cues (Nakamura, 1999).

Major connections of the posterior parietal/anteromedial cortex. Figure 1B shows the main cortical and thalamic inputs to the posterior parietal/anteromedial cortex. The main thalamic input, perhaps the "driver" input (Sherman and Guillery, 2001), comes from two lateral thalamic nuclei. The more lateral cortex, named as areas ParPD and ParR mainly on cyto- and myeloarchitectonic grounds, and that should include extrastriate visual area RL, receives thalamic inputs from the lateroposterior nucleus, less from the laterodorsal nucleus and, to a much smaller degree, from the posterior thalamic nucleus; in addition to a lesser projection from intralaminar thalamic nuclei (central lateral and ventrolateral nuclei) (Giannetti and Molinari, 2002). The more medial area AM, comprising the rostral portion of Oc2MM and $\mathrm{Oc} 2 \mathrm{ML}$, receives its thalamic input from the anterior portion of the lateroposterior thalamic nucleus and adjacent laterodorsal nucleus (Torrealba et al., 1984) and from the posterior thalamic nucleus (Sanderson et al., 1991). These two lateral thalamic nuclei are considered as association relays in that they are not directly connected with ascending sensory inputs or with motor inputs, but are rather polysensory in their responses; some lateral thalamic neurons are in fact head direction cells (Groenewegen and Witter, 2004).

Vestibular input can reach the posterior parietal/anteromedial cortex through several thalamic and cortical relays, but the precise weight of each input has not been explored. A direct projection from the medial vestibular nucleus to the laterodorsal thalamic nucleus has been noted (Shiroyama et al., 1999). In addition, the vestibular cortex sends axonal projections to the parietal association cortex of primates (Guldin et al., 1992), though the existence of an equivalent vestibular cortex has not been determined in the rat. We have noted (Torrealba et al., 1984) direct axonal projections from somatosensory and probably auditory cortices, which may convey vestibular, auditory and propioceptive information to the posterior parietal/anteromedial cortex.

Visual information can also reach the posterior parietal/anteromedial cortex through several pathways. The most likely thalamic input bringing visual input is the lateroposterior nucleus, which in turn gets visual information directly from the retina, from the superior colliculus and from layer $\mathrm{V}$ pyramidal cells of the primary visual cortex. As well, a rather weak but 
consistent projection from the dorsal lateral geniculate nucleus was described (Torrealba et al., 1984). Cortical input with visual content reaches the posterior parietal/ anteromedial cortex via direct corticocortical projections from the primary visual cortex as well as from several extrastriate visual areas in Oc2L (Torrealba et al., 1984) and from two visual areas, one in caudal Oc2M and the other in anterolateral Oc2M, probably area A (see above).

The posterior parietal/anteromedial cortex is strongly and reciprocally connected to two different regions of the limbic (cingulate) cortex. In the midcingulate cortex it is connected to the area $24 \mathrm{~b}$ (frontal eye field), involved in attention. The most likely link between the posterior parietal/anteromedial cortex and the hippocampal formation is via the reciprocal connections between the retrosplenial cortex (posterior cingulate cortex, area 29d), a critical component of the limbic system, and para-hippocampal formation on one hand (Wyss and Van Groen, 1992), and of retrosplenial cortex and the posterior parietal/anteromedial cortex (Vogt and Miller, 1983; Torrealba et al., 1984) on the other hand. These strong connections help to explain the involvement of the posterior parietal/anteromedial cortex in spatial tasks and navigation in concert with the hippocampal formation.

The evidence we have reviewed here, mostly from studies in rats, indicate that the posterior parietal/anteromedial cortex of the rat is a multimodal sensory area, involved in navigation in space, in spatial memory, and in tasks where associations between different sensory modalities are relevant. It is likely that the posterior parietal/ anteromedial cortex of the rat is homologous to at least part of the parietal association cortex of primates, including humans.

\section{ACKNOWLEDGEMENTS}

Financed by Fondecyt 1060476 and Iniciativa Científica Milenio $N^{\circ}$ P06/008-F (F.T.) and by the Pew Latin American Fellows Program in the Biomedical Science (J.L.V.)

\section{REFERENCES}

BYRNE P, BECKER S, BURGESS N (2007) Remembering the past and imagining the future: a neural model of spatial memory and imagery. Psychol.Rev 114: 340375.

CABEZA R, CIARAMELLI E, OLSON IR, MOSCOVITCH M (2008) The parietal cortex and episodic memory: an attentional account. Nat Rev Neurosci 9: 613-625.

CHEN LL, LIN LH, BARNES CA, MCNAUGHTON BL (1994a) Head-direction cells in the rat posterior cortex. II. Contributions of visual and ideothetic information to the directional firing. Exp.Brain Res. 101: 24-34.

CHEN LL, LIN LH, GREEN EJ, BARNES CA, MCNAUGHTON BL (1994b) Head-direction cells in the rat posterior cortex. I. Anatomical distribution and behavioral modulation. Exp.Brain Res. 101: 8-23.

ESPINA-MARCHANT P, PINTO-HAMUY T, BUSTAMANTE D, MORALES P, ROBLES L, HERRERA-MARSCHITZ M (2006) Spatial cognition and memory: a reversible lesion with lidocaine into the anteromedial/posterior parietal cortex (AM/PPC) affects differently working and long-term memory on two foraging tasks. Biol Res. 39: 601-609.

ESPINOZA S, PINTO-HAMUY T, PASSIG C, CARRENO F, MARCHANT F, URZUA C (1999) Deficit in the water-maze after lesions in the anteromedial extrastriate cortex in rats. Physiol Behav. 66: 493-496.

ESPINOZA SG, THOMAS HC (1983) Retinotopic organization of striate and extrastriate visual cortex in the hooded rat. Brain Res. 272: 137-144.

FYHN M, MOLDEN S, WITTER MP, MOSER EI, MOSER MB (2004) Spatial representation in the entorhinal cortex. Science 305: 1258-1264.

GALLARDO L, MOTTLES M, VERA L, CARRASCO MA, TORREALBA F, MONTERO VM, PINTOHAMUY T (1979) Failure by rats to learn a visual conditional discrimination after lateral peristriate cortical lesions. Physiol.Psychol. 7: 173-177.

GIANNETTI S, MOLINARI M (2002) Cerebellar input to the posterior parietal cortex in the rat. Brain Res.Bull. 58: 481-489.

GROENEWEGEN HJ, WITTER MP (2004) Thalamus. In: PAXINOS G (ed) The rat nervous system. Third. San Diego: Elsevier Academic Press. pp: 407-453.

GULDIN WO, AKBARIAN S, GRUSSER OJ (1992) Cortico-cortical connections and cytoarchitectonics of the primate vestibular cortex: a study in squirrel monkeys (Saimiri sciureus). J Comp Neurol. 326: 375401.

HAFTING T, FYHN M, MOLDEN S, MOSER MB, MOSER EI (2005) Microstructure of a spatial map in the entorhinal cortex. Nature 436: 801-806.

HOK V, LENCK-SANTINI PP, ROUX S, SAVE E, MULLER RU, POUCET B (2007) Goal-related activity in hippocampal place cells. J Neurosci 27: 472-482.

HOK V, SAVE E, LENCK-SANTINI PP, POUCET B (2005) Coding for spatial goals in the prelimbic/ infralimbic area of the rat frontal cortex. Proc.Natl.Acad.Sci.U.S.A 102: 4602-4607.

KERR KM, AGSTER KL, FURTAK SC, BURWELL RD (2007) Functional neuroanatomy of the parahippocampal region: the lateral and medial entorhinal areas. Hippocampus 17: 697-708.

KOLB B (1990) Posterior parietal and temporal association cortex. In: KOLB B TEES RC (eds) The cerebral cortex of the rat. Cambridge, Massachusetts: The MIT Press. pp: 459-471. 
KOLB B, BUHRMANN $\mathrm{K}$, MCDONALD $\mathrm{R}$, SUTHERLAND RJ (1994) Dissociation of the medial prefrontal, posterior parietal, and posterior temporal cortex for spatial navigation and recognition memory in the rat. Cereb.Cortex 4: 664-680.

MCDANIEL WF (1985) Functions of the posterior neocortex of the rat. IRCS Med.Sci. 13: 286-289.

MCNAUGHTON BL, BATTAGLIA FP, JENSEN O, MOSER EI, MOSER MB (2006) Path integration and the neural basis of the 'cognitive map'. Nat Rev Neurosci 7: 663-678.

MILLER VM, BEST PJ (1980) Spatial correlates of hippocampal unit activity are altered by lesions of the fornix and endorhinal cortex. Brain Res. 194: 311-323.

MONTERO VM (1993) Retinotopy of cortical connections between the striate cortex and extrastriate visual areas in the rat. Exp.Brain Res. 94: 1-15.

MONTERO VM, BRAVO H, FERNANDEZ V (1973a) Striate-peristriate cortico-cortical connections in the albino and gray rat. Brain Res. 53: 202-207.

MONTERO VM, ROJAS A, TORREALBA F (1973b) Retinotopic organization of striate and peristriate visual cortex in the albino rat. Brain Res. 53: 197-201.

MULLER RU, KUBIE JL (1987) The effects of changes in the environment on the spatial firing of hippocampal complex-spike cells. J Neurosci 7: 1951-1968.

NAKAMURA K (1999) Auditory spatial discriminatory and mnemonic neurons in rat posterior parietal cortex. J Neurophysiol 82: 2503-2517.

O'KEEFE J (1978) The hippocampus as a cognitive map. Clarendon: Oxford.

O'KEEFE J, DOSTROVSKY J (1971) The hippocampus as a spatial map. Preliminary evidence from unit activity in the freely-moving rat. Brain Res. 34: 171-175.

PALOMERO-GALLAHER N, ZILLES K (2004) Isocortex. In: PAXINOS G (ed) The rat nervous system. Third. San Diego: Elsevier Academic Press. pp: 729-757.

PINTO-HAMUY T, MONTERO VM, TORREALBA F (2004) Neurotoxic lesion of anteromedial/posterior parietal cortex disrupts spatial maze memory in blind rats. Behav.Brain Res. 153: 465-470.

PINTO-HAMUY T, OLAVARRIA J, GUIC-ROBLES E, MORGUES M, NASSAL O, PETIT D (1987) Rats with lesions in anteromedial extrastriate cortex fail to learn a visuosomatic conditional response. Behav.Brain Res. 25: 221-231.

QUIRK GJ, MULLER RU, KUBIE JL (1990) The firing of hippocampal place cells in the dark depends on the rat's recent experience. J Neurosci 10: 2008-2017.

SANCHEZ RF, MONTERO VM, ESPINOZA SG, DIAZ E, CANITROT M, PINTO-HAMUY T (1997) Visuospatial discrimination deficit in rats after ibotenate lesions in anteromedial visual cortex. Physiol Behav. 62: 989-994.

SANDERSON KJ, DREHER B, GAYER N (1991)
Prosencephalic connections of striate and extrastriate areas of rat visual cortex. Exp.Brain Res. 85: 324-334.

SAVE E, POUCET B (2005) Piloting. In: WHISHAW IQ KOLB B (eds) The behavior of the laboratory rat. New York: Oxford University Press. pp: 392-400.

SCHAAFSMA SJ, DUYSENS J (1996) Neurons in the ventral intraparietal area of awake macaque monkey closely resemble neurons in the dorsal part of the medial superior temporal area in their responses to optic flow patterns. J Neurophysiol 76: 4056-4068.

SHERMAN SM, GUILLERY RW (2001) Exploring the thalamus. San Diego, USA: Academic Press.

SHIROYAMA T, KAYAHARA T, YASUI Y, NOMURA J, NAKANO K (1999) Projections of the vestibular nuclei to the thalamus in the rat: a Phaseolus vulgaris leucoagglutinin study. J Comp Neurol. 407: 318-332.

STEWARD O (1976) Topographic organization of the projections from the entorhinal area to the hippocampal formation of the rat. J Comp Neurol. 167: 285-314.

STEWARD O, SCOVILLE SA (1976) Cells of origin of entorhinal cortical afferents to the hippocampus and fascia dentata of the rat. J Comp Neurol. 169: 347-370.

THOMAS HC, ESPINOZA SG (1987) Relationships between interhemispheric cortical connections and visual areas in hooded rats. Brain Res. 417: 214-224.

TORREALBA F, OLAVARRIA J, CARRASCO MA (1984) Cortical connections of the anteromedial extrastriate visual cortex in the rat. Exp.Brain Res. 56: 543-549.

VOGT BA, MILLER MW (1983) Cortical connections between rat cingulate cortex and visual, motor, and postsubicular cortices. J Comp Neurol. 216: 192-210.

WALLACE DG, WHISHAW IQ (2005) Dead reckoning. In: WHISHAW IQ KOLB B (eds) The behavior of the laboratory rat. New York: Oxford University Press. pp: 401-409.

WHISHAW IQ, VANDERWOLF CH (1973) Hippocampal EEG and behavior: changes in amplitude and frequency of RSA (theta rhythm) associated with spontaneous and learned movement patterns in rats and cats. Behav.Biol 8: 461-484.

WIENER SL, TAUBE JS (2005) Head direction cells and the neural mechanism of spatial orientation. Cambridge, MA: The MIT press.

WITTER MP, AMARAL DG (2004) Hippocampal formation. In: PAXINOS $G$ (ed) The rat nervous system. Third Ed.. San Diego: Elsevier Academic Press. pp: 635-704.

WYSS JM, VAN GROEN T (1992) Connections between the retrosplenial cortex and the hippocampal formation in the rat: a review. Hippocampus 2: 1-11.

ZILLES K (1990) Anatomy of the neocortex: cytoarchitecture and myeloarchitecture. In: KOLB B TEES RC (eds) The cerebral cortex of the rat. Cambridge, Massachusets: The MIT Press. pp: 77-112. 